\title{
Emotional Wellbeing in Saudi Arabia During the COVID-19 Pandemic: A National Survey
}

This article was published in the following Dove Press journal:

Risk Management and Healthcare Policy

\author{
Abbas Al Mutair (iD ${ }^{1-3}$ \\ Mohammed Alhajji ${ }^{4}$ \\ Abbas Shamsan ${ }^{5}$
}

'Research Center, Almoosa Specialist Hospital, Al-Ahsa, Saudi Arabia; ${ }^{2}$ College of Nursing, Princess Norah University, Riyadh, Saudi Arabia; ${ }^{3}$ School of Nursing, Wollongong University, Wollongong, Australia; ${ }^{4}$ College of Public Health, Temple University, Philadelphia, PA, USA; ${ }^{5}$ Research Center, Dr. Sulaiman Al Habib Medical Group, Riyadh, Saudi Arabia
Correspondence: Abbas Al Mutair Research Center, Almoosa Specialist Hospital, P.O. Box 301578, Dahran St Alfaisal, Al-Ahsa, Riyadh, I I643, Saudi Arabia

Tel +966 II 5259999 Ext 2975

Email Abbas4080@hotmail.com
Purpose: This study aims to evaluate the overall emotional wellbeing and emotional predictors of the Saudi population during COVID-19.

Patients and Methods: A cross-sectional design was employed; the data were collected by using the Arabic version of the Mental Health Inventory.

Results: A total of 5041 participants were successfully recruited over 1 week. The participants scored moderately on Anxiety, Depression, Loss of Behavioral/Emotional Control, General Positive Affect, Emotional Ties, and Life Satisfaction. The results indicated that age, gender, marital status, socioeconomic status, and having chronic health conditions are major predictors of emotional wellbeing during the COVID-19 pandemic.

Conclusion: A rehabilitation program should be initiated to restore the community function and the wellbeing of individuals who have been impacted by the COVID-19 pandemic.

Keywords: emotional wellbeing, anxiety, depression, COVID-19, Saudi Arabia

\section{Introduction}

The novel coronavirus outbreak had occurred in Wuhan (Hubei, China) in December 2019 and evolve rapidly throughout the world. The World Health Organization (WHO) characterized COVID-19 as a pandemic in March 2020. This crisis has caused immense stress on governments, institutions, and the world population. Delirium, psychosis, severe anxiety, and depression have been well observed during this pandemic. ${ }^{1}$ The Centers for Disease Control and Prevention (CDC) reported that pandemics are marked by disrupted sleep cycles, concentration difficulty, fear and excessive worry about one's own life and their loved ones', and increased substance abuse. ${ }^{2}$ For those reasons, it is essential to assess the mental health status during and after pandemics to provide new resources and interventions. $^{3}$

Pandemics, such as COVID-19, can be mentally demanding due to the unpredictability of the situation, the uncertainty of when to control the disease, and the seriousness of the risk. These, along with misinformation and exaggerating media coverage can put immense mental pressure on individuals and societies. ${ }^{1}$ In a study measuring the psychological impact of COVID-19 in China, 26\% reported that they suffered from mild to moderate depression, $4.3 \%$ had severe depression, and over one third reported moderate to severe anxiety. ${ }^{4}$ People with families and children tend to have a higher level of stress. ${ }^{4}$ Another survey in China reported that COVID-19 has caused high levels of emotional stress. ${ }^{5}$ Additionally, Gavidia illustrates that $88 \%$ of employees in an American sample reported moderate to 
extreme stress levels during COVID- $19 .{ }^{6}$ Similarly, the heightened sense of concerns and stress have been also reportedly experienced by healthcare workers during the MERS-CoV outbreak in Saudi Arabia, and during the severe acute respiratory syndrome (SARS) outbreak. ${ }^{7-10}$

While these studies and surveys have assessed the mental health impact of MERS-CoV and COVID-19 in different countries, to our knowledge, there has not been any national assessment done on the Saudi population with regards to COVID-19. Given the cultural variations in responding to natural disasters and pandemics, it is essential to understand how Saudis particularly react to this unprecedented pandemic in order to assist government agencies and organizations in safeguarding the psychological wellbeing of the society. ${ }^{11}$ This national, crosssectional study aims to evaluate the emotional wellbeing of the general population in Saudi Arabia.

\section{Patients and Methods}

\section{Participants}

An online link of the study was posted on social media including Twitter, Facebook, Telegram and WhatsApp across Saudi Arabia to invite people to complete the survey through Qualtrics. Qualtrics is an online survey tool that allowed us to build, distribute and analyse the survey responses. Ethics approval to conduct the study was obtained from Dr. Sulaiman Al Habib Medical Group - Institutional Review Board (RC20.03.78), and this study was conducted in accordance with the Declaration of Helsinki. This was an anonymous survey that did not collect any personal details about the participants and participation was voluntary, hence consent was implied. A probability convenience sample method was employed to recruit the study participants. The convenience sample technique was considered as the most appropriate method to reach the maximum population and to enable us to explore the study phenomena and acquire information about the study area. The sampling method used was intended to allow an equal opportunity to the population to be enrolled in the study, increase the representativeness of the sample, and decrease sampling error and bias. Several measures have been taken to control biases that might arise from the sample criteria. The criteria included: 1) Living in Saudi Arabia and 18 years of age or older, 2) with no previous mental health diagnosis, and 3) can read and write Arabic.
Several measures were taken to maximize the power estimation of the current study which included medium effect size, and significance level (alpha) which was set at $0.05 \%$ and $80 \%$ power. This was undertaken to allow the probability of the statistical test to detect the differences or relationships in the study population. The effect size referred to the degree to which the null hypothesis was not true, while the significance level was set at 0.05 so there would be only a $5 \%$ probability of getting Type I error. ${ }^{12}$ According to Munro (2005), medium effect size between 0.3 and 0.5 is suitable for well-defined theoretical framework studies such as the current study as many relevant studies have been conducted in the same area. ${ }^{13-16}$ Additionally, $0.80(80 \%)$ is considered the best acceptable power for a study according to Cohen. ${ }^{17}$ Therefore, to allow estimation of moderate size effect $(f$ $=0.3$ ) at a $5 \%$ significance level with $80 \%$ power, a sample size of 5000 was determined using $\mathrm{G}^{*}$ Power.

\section{Measures}

The emotional wellbeing has been measured by using the Arabic version of the Mental Health Inventory (AV-MHI -38). ${ }^{15}$ The original scale (MHI-38) is an extensively validated scale that has been developed and used with multiple populations in medical outcomes survey projects to measure aspects of mental wellbeing, including anxiety and depression. ${ }^{13,14}$ The MHI has 38 items divided into 6 domains, including Anxiety, Depression, Loss of Behavioral/Emotional Control, General Positive Affect, Emotional Ties, and Life Satisfaction. Using a cross-cultural multicenter survey approach, a validation study among Arabic-speaking nursing students to examine the psychometric testing of the AV-MHI-38 has shown good internal consistency with Cronbach's $\alpha=0.85$ and acceptable reliability measures. ${ }^{15}$

All items are scored on a 6-point Likert scale (range 1-6) except two items scored on a 5-point Likert scale (range 1-5). The Anxiety subscale includes nine items with a possible score range between 9 and 54, in which higher scores indicate greater anxiety. The Depression subscale includes five items with a score range between 4 and 23, in which higher scores indicate greater depression. Loss of Behavioral/Emotional Control subscale includes nine items ranging from 9 to 53, with higher scores revealing a greater loss of behavioral/emotional control. In the General Positive Affect subscale, there are 10 items with a score ranging from 10 to 60 , with higher scores indicating greater positive affect. The 
Emotional Ties and Life Satisfaction subscales have two items and one item, respectively, with scores ranging from 2 to 12 and 1 to 6 , in which higher scores indicate stronger emotional ties and greater life satisfaction, respectively.

Additionally, the MHI may be aggregated into three global scales, which represent complementary summary scores. First, the Psychological distress global scale is composed by summing the scores of 24 items, with a score range from 24 to 142 . Second, the Psychological wellbeing global scale is calculated by summing the scores of 14 items, with a possible score range from 14 to 84 . There is no item overlap between these two global scales. Finally, the raw score range of the Mental Health Index is 38-226. MHI-38 is a single score based on all 38 items designed as a high-level summary index of the person's mental wellbeing; higher scores on the Mental Health Index indicate greater psychological wellbeing and relatively less psychological distress. ${ }^{13}$ Socio-demographic data were collected to capture participants' age, gender, marital status, education, perceived socioeconomic status, citizenship, mental health diagnosis, chronic health conditions, and levels of exposure to COVID-19.

\section{Data Analysis}

Several items were reverse-scored to ensure that higher scores reflect the global scales and subscales as per the MHI-38 Scoring System and Procedure. ${ }^{18}$ One item from the original AV-MHI-38, Q13, a component of the anxiety subscale, was inadvertently excluded in the data collection phase due to a technical error in the survey platform. To mitigate the issue and to maintain the scoring and validity of the scale, this item was assigned the average score (mean $=3.7$ ) which was found in two previous studies that used Saudi samples. ${ }^{15,16}$ This method was preferred over mean imputation methods as the latter resulted in highly biased estimates when more than $10 \%$ of subjects had missing data. ${ }^{19}$ Descriptive statistics, subscales, and global scales were calculated per the MHI-38 Scoring System and Procedure. ${ }^{18}$

Backward multiple linear regression was used to find the best fitting model to identify the socio-demographic predictors of emotional wellbeing. The normality of the data was confirmed using Q-Q normality tests and histograms for all scales. All assumptions of multiple linear regression were confirmed prior to the analysis. ${ }^{20}$ All analysis was conducted using the IBM Statistical Package for the Social Sciences statistical software package version for Mac, version 26.0; a p-value $<0.05$ was considered statistically significant.

\section{Results}

A total of 5041 participants' responses were collected and the majority of the sample $(67.8 \%)$ was female. Participants' ages ranged from 18 to 66 years, with an average age of 29.8 years $(\mathrm{SD}=8.1)$. For education, 3784 $(75.06 \%)$ reported to have an education higher than high school. Furthermore, only 4\% reported "poor" perceived socioeconomic status. Slightly over half of all participants, $204(51.4 \%)$ were single at the time of the survey. The overwhelming majority of $4641(92.1 \%)$ did not have any chronic health conditions to report. Only 1008 (20\%) reported that they lived with someone whose job entailed working with patients. The demographic characteristics of the sample are shown in Table 1.

The AV-MHI-38 results showed moderate levels of anxiety, depression, and loss of behavioral/emotional control. As seen in Table 2, the mean of each subscale is noted to fall at approximately half-way of its possible range. For instance, the depression subscale has a mean of $11.8(\mathrm{SD}=$ 7.1) and a possible range of 4-23.

Table 3 is reflective of Table 2 in that it demonstrates moderate levels of each domain. All three global scales have means in the middle point of the respective range. For example, the Psychological Distress scale has a mean of $75.5(\mathrm{SD}=15.4)$ with a possible range of 24-142.

\section{Predicting Emotional Wellbeing}

Since backward stepwise regression models were used to predict emotional wellbeing as measured by the two global scales (Psychological Distress and Psychological Wellbeing) and the MHI-38, the three models differed slightly on the constituent socio-demographic predictors. However, the five variables of age, gender, marital status, socioeconomic status, and chronic health conditions status were present in all three final optimum models. These explanatory independent variables had $p$-values lower than 0.05 across the three models. The other sociodemographic variables of citizenship, administrative regions, being formally diagnosed with a mental illness, and working in a job with direct patient contact had $p$-values that exceeded 0.05 in all models, and thus they were automatically excluded in the final fitted models. Table 4 presents the results of the regression analyses for Psychological Distress, Psychological Wellbeing, and the Mental Health Index. 
Table I Demographics of the Total Sample

\begin{tabular}{|c|c|}
\hline \multicolumn{2}{|l|}{ Demographic Attributes } \\
\hline Total number of participants & $N=504 I$ \\
\hline \multirow[t]{2}{*}{ Mean age in years (SD, Range) } & $29.8(8.6,18-66)$ \\
\hline & n (\%) \\
\hline \multicolumn{2}{|l|}{ Gender } \\
\hline Males & $1621(32.2)$ \\
\hline Females & $3420(67.8)$ \\
\hline \multicolumn{2}{|l|}{ Socioeconomic status } \\
\hline Poor & $204(4)$ \\
\hline Good & 1906 (37.8) \\
\hline Very good & $226 \mid(44.9)$ \\
\hline Excellent & $670(13.3)$ \\
\hline \multicolumn{2}{|l|}{ Education } \\
\hline High school or less & $1257(24.9)$ \\
\hline Associate degree/Vocational & $415(8.2)$ \\
\hline Bachelor's & $2853(56.6)$ \\
\hline Master's & $430(8.5)$ \\
\hline $\mathrm{PhD}$ & $86(1.7)$ \\
\hline \multicolumn{2}{|l|}{ Citizenship } \\
\hline Saudis & $4829(95.8)$ \\
\hline Non-Saudis & $212(4.2)$ \\
\hline \multicolumn{2}{|l|}{ Administrative regions } \\
\hline Eastern & $2079(4 I .2)$ \\
\hline Riyadh & $1278(25.4)$ \\
\hline Makkah & $727(14.4)$ \\
\hline Other & $957(19)$ \\
\hline \multicolumn{2}{|l|}{ Formally diagnosed with a mental illness } \\
\hline Yes & $403(8)$ \\
\hline No & $44,638(92)$ \\
\hline \multicolumn{2}{|l|}{ Having a chronic health condition } \\
\hline Yes & $400(7.9)$ \\
\hline No & $464 I(92.1)$ \\
\hline \multicolumn{2}{|l|}{ Job entails working with patients directly } \\
\hline Yes & $596(11.8)$ \\
\hline No & $2914(57.8)$ \\
\hline Not applicable & I53| (30.4) \\
\hline \multicolumn{2}{|l|}{$\begin{array}{l}\text { Live with someone who works with } \\
\text { patients }\end{array}$} \\
\hline Yes & $1008(20)$ \\
\hline No & $4033(80)$ \\
\hline
\end{tabular}

For instance, a significant regression equation was found to predict Psychological Distress $(F(7,5033)=$ $119.4, p<0.0001)$, with an adjusted $\mathrm{R}^{2}$ of 0.14 . In all models, age was measured in years, gender was coded
Table 2 Summary of the Six Mental Health Index Subscales

\begin{tabular}{|l|c|c|c|}
\hline Subscale & $\begin{array}{c}\text { Possible } \\
\text { Range }\end{array}$ & Mean & $\begin{array}{c}\text { Standard } \\
\text { Deviation }\end{array}$ \\
\hline Anxiety & $9-54$ & 32.4 & 7.1 \\
\hline Depression & $4-23$ & 11.8 & 4.2 \\
\hline $\begin{array}{l}\text { Loss of behavioral/ } \\
\text { emotional control }\end{array}$ & $9-53$ & 24.3 & 7.2 \\
\hline General positive affect & $10-60$ & 28.8 & 8.5 \\
\hline Emotional ties & $2-12$ & 5.3 & 2.3 \\
\hline Life satisfaction & $1-6$ & 2.6 & 1.2 \\
\hline
\end{tabular}

Table 3 Summary of Global Scales and the Mental Health Index

\begin{tabular}{|l|c|c|c|}
\hline Scale & $\begin{array}{c}\text { Possible } \\
\text { Range }\end{array}$ & Mean & $\begin{array}{c}\text { Standard } \\
\text { Deviation }\end{array}$ \\
\hline Psychological Distress & $24-142$ & 75.5 & 15.4 \\
\hline Psychological Wellbeing & $14-84$ & 40.7 & 10.4 \\
\hline $\begin{array}{l}\text { Mental Health Index } \\
\text { (MHI-38) }\end{array}$ & $38-226$ & 116.2 & 23.6 \\
\hline
\end{tabular}

as $1=$ male, 2 = female, marital status was coded as $1=$ single, 2 = married, and $3=$ divorced or widowed, education and SES were coded in increasing order, having a chronic illness was coded as $1=$ yes, $2=$ no, and home exposure was coded as $1=$ yes, $2=$ no. Participants' Psychological Distress decreased by 0.03 units for each level of education and 0.20 for each year of age. All predictors in these fitted models were significant.

\section{Discussion}

This study aimed to measure predictors of emotional wellbeing during the COVID-19 pandemic in Saudi Arabia. It measured the levels of anxiety, depression, behavioral control, positive affect, and general distress, so as to design strategies to alleviate and rehabilitate during this pandemic. Our findings suggest that Saudis have been found to be quite resilient to COVID-19 stress in comparison to other countries experiencing this pandemic. The data collected are somewhat surprising and counterintuitive in some aspects. It leads to the possibility of further investigation into the minds of the Saudi population to further understand what has and is contributing to their resilience. 
Table 4 Regression Models Predicting Emotional Wellbeing $(N=504 \mathrm{I})$

\begin{tabular}{|c|c|c|c|c|c|c|c|}
\hline Scale & $\mathbf{t}$ & $\mathbf{p}$ & B & $\mathbf{F}$ & df & $\mathbf{p}$ & Adj. R2 \\
\hline Psychological Distress & & & & 119.4 & 7,5033 & $<0.0001$ & 0.14 \\
\hline Age & -12.2 & $<0.0001$ & -0.20 & & & & \\
\hline Female & 11.8 & $<0.0001$ & 0.16 & & & & \\
\hline Marital status & -6.5 & $<0.0001$ & -0.10 & & & & \\
\hline Education & -2.2 & 0.025 & -0.03 & & & & \\
\hline SES & -12.9 & $<0.0001$ & -0.17 & & & & \\
\hline Chronic illness & -2.5 & 0.012 & -0.03 & & & & \\
\hline Home exposure & -4.2 & $<0.0001$ & -0.05 & & & & \\
\hline Psychological Wellbeing & & & & 107.4 & 5,5035 & $<0.0001$ & 0.095 \\
\hline Age & -7.6 & $<0.0001$ & -0.13 & & & & \\
\hline Female & 4.6 & $<0.0001$ & 0.06 & & & & \\
\hline Marital status & -7.8 & $<0.0001$ & -0.13 & & & & \\
\hline SES & -15.6 & $<0.0001$ & -0.2 & & & & \\
\hline Chronic illness & -2.4 & 0.016 & -0.03 & & & & \\
\hline Mental Health Index & & & & I37.4 & 6,5034 & $<0.0001$ & 0.14 \\
\hline Age & -11.8 & $<0.0001$ & -0.19 & & & & \\
\hline Female & 9.7 & $<0.0001$ & 0.13 & & & & \\
\hline Marital status & -7.8 & $<0.0001$ & -0.12 & & & & \\
\hline SES & -15.6 & $<0.0001$ & -0.20 & & & & \\
\hline Chronic illness & -2.8 & $<0.0001$ & -0.04 & & & & \\
\hline Home exposure & -3.5 & $<0.0001$ & -0.05 & & & & \\
\hline
\end{tabular}

Notes: $\beta$, standardized. Home Exposure, living with one who works with patients.

Abbreviation: SES, socioeconomic status.

As this pandemic continues to rage, people are worried about getting infected or a loved one getting infected. Also, death numbers and the media coverage that handling the situation are potential factors that might contribute to increase the levels of anxiety and depression. However, loss of behavioral and/or emotional control can be frequent at moments such as COVID-19. In China, one study found that a low perception of being infected is significantly associated with low stress and vice versa. ${ }^{4}$ This is supported by another 2010 H1N1 study done in Hong Kong that was able to correlate peoples' fears for either themselves or loved ones contracting the virus, which affected their threat level and therefore their emotional stress response. This Hong Kong study was focused on emotional stress levels and avoidance behaviors. It was conducted during the initial phase of the H1N1 pandemic in 2010. The study concluded that lack of adequate, clear, and concise information contributed greatly to increased emotional distress and behaviors of avoidance. Evidently, the stages of a pandemic can greatly affect emotional stress responses because of the ties to public health education levels. Concerns can be calmed when the affected population receives education about the pandemic threat including transmission, at-risk populations, symptoms, treatment, and interventional steps to take to lessen the spread and severity of the new disease. ${ }^{21}$

In this Saudi-based study, results have shown low levels of life satisfaction, general positive affect, and emotional ties. Similar conclusions were found in China, where life satisfaction was found to be lower during COVID-19, and people presented more negative emotions. ${ }^{22}$ However, research shows that positive feelings increase over time during natural disasters and pandemics ${ }^{23,24}$ due to prosocial behaviors, such as social solidarity which produces higher group cohesiveness. ${ }^{23-25}$ During COVID-19 in Saudi Arabia, positive affect and emotional ties were mild since the government made it clear that the public's health was the priority during the crisis, which may have contributed to reports of moderate life satisfaction levels. Psychological Distress, psychological wellbeing, and the Mental Health Index (MHI-38) scores were mainly moderate. Given the circumstances in Saudi Arabia, where there was an early implementation of social distancing, as the governmental focus was mainly on the public health and economic status. Make note that the Kingdom of Saudi Arabia is a monarchy and its 
citizenry has not only common religious ties but also tribal ties as well as large traditional family bonds. Saudi Arabia has high literacy levels, universal healthcare that is at par with western countries, and a large interactive social media presence. This results in a high regard for authority and the ability to communicate and disseminate information easily, which then leads to high compliance with proposed preventative measures.

The results of the current study indicate that age, gender, marital status, and socioeconomic status are major predictors of emotional wellbeing during the COVID-19 pandemic in Saudi Arabia. Elderly individuals, females and married persons had higher psychological distress compared to the rest of the study participants. Female, elderly, and married individuals reported higher anxiety, depression, and loss of behavioral/emotional control. A possible interpretation here is that elderly and female individuals may perceive the pandemic as having less psychological control which makes them less able to control their emotions resulting in higher levels of depression, anxiety, and psychological distress. Similar findings were indicated in a study where gender was a predictor of psychological distress, showing that the male gender is significantly associated with lower stress. ${ }^{4}$ Another study found that gender, social support, and specific experiences were factors for psychological distress. ${ }^{26}$ While a review of the literature makes it apparent that being informed, having solid communication and a feeling of governmental control over the situation is also deemed very important.

Various administrative departments in Saudi Arabia responded to control COVID-19 transmission. The government allocated resources from different ministries and governmental and non-governmental departments to stabilize the people's livelihood and comforting the domestic society and ensuring information transparency. Though the spread of COVID-19 will continue for a period of time and the psychological impacts of the pandemic might be greater in the next two years.

The cultural response to natural disasters and pandemics varies from one society to another. ${ }^{11}$ Such crises stem from psychological problems to particular individuals with more vulnerability to develop mental illnesses. An abundance of vulnerable groups have been identified in the literature, such as elderly people, pregnant women, international students, migrant employees, homeless individuals, and history of having mental illnesses. ${ }^{27-32}$ This study is endeavoring to help understand the Saudi society's response to the pandemic and crises. A plethora of quantitative and qualitative studies must be conducted in the future to fill the knowledge gap in educating and increasing the public awareness on responding to pandemics, providing recommendations for interventions and prevention, and suggesting therapeutic provisions and strategies.

Possible limitations for the current study include the cross-sectional study design as a longitudinal design would demonstrate whether the effect will continue. The limitations also relate to self-reporting and the fact that the surveys were distributed online mostly through a social media platform. These platforms are mainly accessed by the younger generation and this might have been the reason most of the respondents' age was around 30 years. In addition, self-reporting may be influenced by other factors, such as participants wanting to appear more informed than they are. Furthermore, gaps in the perception of knowledge levels of folks participating in self-reporting must be considered.

For future studies, data should be collected on different phases of the pandemic as the 2010 Hong Kong study demonstrated. ${ }^{21}$ Capturing data at different points will also demonstrate the length of time it takes to start the positive feelings that build during a pandemic when feelings of community and being in it together materialize. Furthermore, there should be qualitative studies to assess psychological and emotional wellbeing. Qualitative studies can provide the why behind quantitative study results. As such, they can uncover the depth of reasoning behind forced answer questions and provide valuable information that can be applied in future quantitative studies. Qualitative studies have an important role to fill. And as such, there should be a considered approach, especially in studies involving personal feelings and perceptions.

\section{Conclusion}

The overall emotional wellbeing of the Saudi population during the COVID-19 pandemic is classified as moderate. The emotional wellbeing predictors found through measuring the Saudis level of anxiety, depression, and behavioral control, positive affect and general distress were age, gender, and very good socioeconomic status. The mean of the psychological distress was 75.5, while the mean of the psychological wellbeing was 40.7 , and the mental health index mean was 116.2. Likely, the moderate level of the overall emotional wellbeing of the Saudi population during COVID-19 was positively affected by the timely and well-orchestrated response of the Saudi Arabian health authorities and government agencies. 
The pre-emptive and great strides taken during the COVID-19 pandemic by countries like Saudi Arabia provide a starting point from which to build upon. However, current findings also revealed different aspects of emotional and mental wellbeing that are being negatively affected during the COVID-19 pandemic among the general Saudi population. Interventions, such as rehabilitation programs that address concerns associated with pandemics, may be a way to restore the community functions and wellbeing of individuals who have been impacted by the COVID-19 pandemic in Saudi Arabia.

In conclusion, when informed populations have confidence in their leaders, and feel like they are being taken care of, as valued members of their community/country, they are much less likely to have high-stress levels during pandemic situations. This is made clear by this study's results and supported by the previously published studies. All of the findings can be used to improve mental health status and psychological resilience for future similar infectious disease outbreaks and pandemics.

\section{Data Sharing Statement}

The data used to support the findings of this study are available from the corresponding author upon request.

\section{Acknowledgments}

The authors would like to thank Dr. Abdul Rehman Zia Zaidi for editing and reviewing this manuscript and Dr. Sulaiman Al-Habib Medical Group and their Research Center for their tremendous support.

\section{Disclosure}

The authors report no conflicts of interest in this work.

\section{References}

1. World Health Organization. Mental health and psychosocial considerations during the COVID-19 outbreak; 2020. Available from: https:// www.who.int/docs/default-source/coronaviruse/mental-healthconsiderations.pdf. Accessed March 31, 2020.

2. Centers for Disease Control and Prevention. Coping with stress; 2020. Available from: https://www.cdc.gov/coronavirus/2019-ncov/daily-life -coping/managing-stress-anxiety.html. Accessed February 27, 2021.

3. Lee SM, Kang WS, Cho A-R, Kim T, Park JK. Psychological impact of the 2015 MERS outbreak on hospital workers and quarantined hemodialysis patients. Compr Psychiatry. 2018;87:123-127. doi:10.1016/j.comppsych.2018.10.003

4. Wang C, Pan R, Wan X, et al. Immediate psychological responses and associated factors during the initial stage of the 2019 coronavirus disease (COVID-19) epidemic among the general population in China. Int $J$ Environ Res Public Health. 2020;17(5):1729. doi:10.3390/ijerph17051729
5. Wang H, Xia Q, Xiong Z, et al. The psychological distress and coping styles in the early stages of the 2019 coronavirus disease (COVID-19) epidemic in the general mainland Chinese population: a web-based survey. PLoS One. 2020;15(5):e0233410. doi:10.1371/ journal.pone. 0233410

6. Gavidia M. How has COVID-19 affected mental health, severity of stress among employees? 2020. Available from: https://www.ajmc. com/newsroom/how-has-covid19-affected-mental-health-severity-ofstress-among-employees. Accessed February 27, 2021.

7. Abolfotouh MA, AlQarni AA, Al-Ghamdi SM, Salam M, Al-Assiri MH, Balkhy HH. An assessment of the level of concern among hospital-based health-care workers regarding MERS outbreaks in Saudi Arabia. BMC Infect Dis. 2017;17(1):4. doi:10.1186/s12879-016-2096-8

8. Maunder R, Hunter J, Vincent L, et al. The immediate psychological and occupational impact of the 2003 SARS outbreak in a teaching hospital. CMAJ. 2003;168(10):1245-1251.

9. Liu T, Chen X, Miao G, Zhang L, Zhang Q, Cheung T. Recommendations on diagnostic criteria and prevention of SARS-related mental disorders. J Clin Psychol Med. 2003;13 (3):188-191.

10. Xiang Y-T, Yang Y, Li W, et al. Timely mental health care for the 2019 novel coronavirus outbreak is urgently needed. Lancet Psychiatry. 2020;7(3):228-229. doi:10.1016/S2215-0366(20)30046-8

11. Gelfand MJ, Raver JL, Nishii L, et al. Differences between tight and loose cultures: a 33-nation study. Science. 2011;332(6033):1100. doi:10.1126/science. 1197754

12. Munro BH. Statistical Methods for Health Care Research. Vol. 1. lippincott williams \& wilkins; 2005.

13. Veit CT, Ware JE. The structure of psychological distress and well-being in general populations. J Consult Clin Psychol. 1983;51 (5):730. doi:10.1037/0022-006X.51.5.730

14. Moxham LJ, Fernandez R, Kim B, Lapkin S, Ten Ham-Baloyi W, Al Mutair A. Employment as a predictor of mental health, psychological distress, anxiety and depression in Australian pre-registration nursing students. $\quad J$ Prof Nurs. 2018;34(6):502-506. doi:10.1016/j. profnurs.2018.03.005

15. Al Mutair A, Al Mohaini M, Fernandez R, Moxham L, Lapkin S, Ham-Baloyi WT. Psychometric testing of the mental health inventory in an Arabian context: cross-cultural validation study. Nurs Open. 2018;5(3):376-383. doi:10.1002/nop2.149

16. Fernandez R, Ham-Baloyi W, Al Mutair A, Lapkin S, Moxham L, Tapsell A. Similarities and differences in well-being between Australian, Saudi Arabian and South African pre-registration nursing students. Collegian. 2020;27(4):416-421. doi:10.1016/j. colegn.2019.11.001

17. Cohen J. Statistical power analysis. Curr Dir Psychol Sci. 1992;1 (3):98-101. doi:10.1111/1467-8721.ep10768783

18. Davies AR, Sherbourne CD, Peterson J, Ware JE. Scoring Manual, Adult Health Status and Patient Satisfaction Measures Used in RAND's Health Insurance Experiment. Santa Monica, California: RAND Corporation; 1988.

19. Eekhout I, de Vet HCW, Twisk JWR, Brand JPL, de Boer MR, Heymans MW. Missing data in a multi-item instrument were best handled by multiple imputation at the item score level. $J$ Clin Epidemiol. 2014;67(3):335-342. doi:10.1016/j.jclinepi.2013.09.009

20. Williams M, Gomez Grajales C, Kurkiewicz D. Assumptions of multiple regression: correcting two misconceptions. Pract Assess Res Eval. 2013;18.

21. Lau JTF, Griffiths S, Choi KC, Tsui HY. Avoidance behaviors and negative psychological responses in the general population in the initial stage of the H1N1 pandemic in Hong Kong. BMC Infect Dis. 2010;10(1):139. doi:10.1186/1471-2334-10-139

22. Li S, Wang Y, Xue J, Zhao N, Zhu T. The impact of COVID-19 epidemic declaration on psychological consequences: a study on active Weibo users. Int J Environ Res Public Health. 2020;17 (6):2032. doi:10.3390/ijerph17062032 
23. Mortensen CR, Becker DV, Ackerman JM, Neuberg SL, Kenrick DT. Infection breeds reticence: the effects of disease salience on self-perceptions of personality and behavioral avoidance tendencies. Psychol Sci. 2010;21(3):440-447. doi:10.1177/0956797610361706

24. Schaller M, Murray DR. Pathogens, personality, and culture: disease prevalence predicts worldwide variability in sociosexuality, extraversion, and openness to experience. J Pers Soc Psychol. 2008;95 (1):212-221. doi:10.1037/0022-3514.95.1.212

25. Tziner A. Group cohesiveness: a dynamic perspective. Soc Behav Personality Int j. 1982;10(2):205-211. doi:10.2224/ sbp.1982.10.2.205

26. Brooks SK, Webster RK, Smith LE, et al. The psychological impact of quarantine and how to reduce it: rapid review of the evidence. Lancet. 2020;395(10227):912-920.

27. Yang Y, Li W, Zhang Q, Zhang L, Cheung T, Xiang Y-T. Mental health services for older adults in China during the COVID-19 outbreak. Lancet Psychiatry. 2020;7(4):e19. doi:10.1016/S22150366(20)30079-1
28. Rashidi Fakari F, Simbar M. Coronavirus pandemic and worries during pregnancy; a letter to editor. Arch Acad Emerg Med. 2020;8 (1):e21-e21

29. Zhai Y, Du X. Mental health care for international Chinese students affected by the COVID-19 outbreak. Lancet Psychiatry. 2020;7(4): e22. doi:10.1016/S2215-0366(20)30089-4

30. Liem A, Wang C, Wariyanti Y, Latkin CA, Hall BJ. The neglected health of international migrant workers in the COVID-19 epidemic. Lancet Psychiatry. 2020;7(4):e20. doi:10.1016/S2215-0366(20) 30076-6

31. Tsai J, Wilson M. COVID-19: a potential public health problem for homeless populations. Lancet Public Health. 2020;5(4):e186-e187. doi:10.1016/S2468-2667(20)30053-0

32. Zhu Y, Chen L, Ji H, Xi M, Fang Y, Li Y. The risk and prevention of novel coronavirus pneumonia infections among inpatients in psychiatric hospitals. Neurosci Bull. 2020;36(3):299-302. doi:10.1007/ s12264-020-00476-9
Risk Management and Healthcare Policy

\section{Publish your work in this journal}

Risk Management and Healthcare Policy is an international, peerreviewed, open access journal focusing on all aspects of public health, policy, and preventative measures to promote good health and improve morbidity and mortality in the population. The journal welcomes submitted papers covering original research, basic science, clinical \& epidemiological studies, reviews and evaluations,
Dovepress

guidelines, expert opinion and commentary, case reports and extended reports. The manuscript management system is completely online and includes a very quick and fair peer-review system, which is all easy to use. Visit http://www.dovepress.com/testimonials.php to read real quotes from published authors. 